\title{
Psychological safety, the hidden curriculum, and ambiguity in medicine
}

\author{
Karina D. Torralba ${ }^{1}$ (D) - Donna Jose ${ }^{1} \cdot$ John Byrne ${ }^{1,2}$ \\ Received: 4 November 2019 / Revised: 4 November 2019 / Accepted: 9 December 2019/Published online: 4 January 2020 \\ (C) International League of Associations for Rheumatology (ILAR) 2020
}

\begin{abstract}
Psychological safety is a feeling that individuals are comfortable expressing and being themselves, as well as comfortable sharing concerns and mistakes without fear of embarrassment, shame, ridicule, or retribution. It has long been recognized as part of successful patient safety and quality improvement processes. However, in the realm of medical education, psychological safety is a relatively unknown concept to many educators and learners alike. Learners, whether students or postgraduate trainees, are in a phase of cognitive apprenticeship whereby they learn not only skills and knowledge from teachers as part of an explicit and formal curriculum. At the same time, a hidden curriculum is also part of the learning environment in the form of norms, values, and behaviors exhibited by teachers. These norms, values, and behaviors become part of the culture of the clinical learning environment. The vulnerability of learners in this environment is magnified by the hierarchal nature of medicine, and the complexity, uncertainty, and the ambiguity inherent to medical conditions. This is especially true of cognitive specialties such as rheumatology. Educators who engage in unprofessional behaviors that result in learner humiliation and shame may serve to dampen productive discourse and scientific dialog. Therefore, educators must embrace psychological safety to foster learning and facilitate high-performing teams in the clinical learning environment.
\end{abstract}

\section{Key Points}

- Psychological safety improves communication and teamwork by allowing individuals to be comfortable expressing and being themselves, as well as comfortable sharing concerns and mistakes without fear of embarrassment, shame, ridicule, or retribution.

- Commonly studied in the context of patient safety and quality improvement, psychological safety should extend towards medical education particularly in the context of allowing medical students and postgraduate trainees to be able to voice clinical reasoning in the face of ambiguity.

- Educators take on a leadership role when having learners under their supervision; as leaders, educators are the prime movers of psychological safety

- Learners in the process of developing their self-identity in the context of their chosen profession adopt not only knowledge and skills within the

framework of an explicit and formal curriculum but also norms and values from daily behavior and language educators present in the clinical learning environment of learners; these norms and values are collectively part of the hidden curriculum.

Keywords Ambiguity $\cdot$ Cognitive apprenticeship $\cdot$ Hidden curriculum $\cdot$ Medical education $\cdot$ Psychological safety

\section{Case review}

Part of the Topical Collection entitled 'Empowering Medical Education to Transform: Learnings from an international perspective'

Karina D. Torralba

ktorralba@1lu.edu

1 Department of Medicine, Division of Rheumatology, Loma Linda University School of Medicine, 11234 Anderson St, MC 1519, Loma Linda, CA 92373, USA

2 Loma Linda Health Care System, Loma Linda, CA, USA

\section{Case}

The setting is the intensive care unit at a level one-trauma center that often receives patients from other hospitals for tertiary care. The senior resident of the team, Dr. Ivy Wong (not her real name), has completed her 28-h shift and is ready to handoff the patients she had cross-covered overnight. Dr. Wong had managed an intubated patient that was transferred from an outside facility for active lupus. Due to concern for lupus flare causing organ damage, the resident paged the Rheumatology 
fellow and attending to discuss the case. She also contacted the Nephrology team due to worsening renal function.

Dr. Wong proceeded to present the case to the team. Her attending had a reputation of having a temper and harshly criticizing residents instead of using constructive criticism. When Dr. Wong mentioned that she had contacted both Rheumatology and Nephrology overnight, the attending questioned why she had done so, asking "So you called them because you did not know what to do?" The attending also asked, "Are you really in your second year of residency? Do you think you should be a senior?" Dr. Wong felt humiliated in front of the team of residents, fellows, students, nurses, and family member who were present. She felt that the attending insinuated that she did not have the knowledge base to manage the patient on her own. Although most of the team members knew the harsh criticism was unwarranted, no one said anything for fear of retaliation. Intimidated and fearful, the team did not dare to speak their minds or bring up differentials on their patients due to fear of being humiliated like Dr. Wong.

The Dilemma People are motivated to protect themselves from embarrassment, ridicule, and shame by not offering ideas, staying quiet, not asking questions, or by withholding from challenging the status quo. Psychologists refer to this risk avoidance as impression management [1-3]. In the clinical learning environment, students and residents have motivations for self-protection including impressing evaluators, striving for independence, and establishing professional credibility. Dr. Wong took on interpersonal risk for the sake of her patient's welfare. By conferring with other specialties, she acknowledges the need for interdisciplinary collaborative care, but also acknowledges limitations to her own breadth of knowledge. However, instead of being recognized for trying to improve patient care, she received ridicule from an individual in power. Observers to the incident similarly face issues related to impression management. When an opportunity arises within a team to speak up, offer a suggestion, ask a question, disagree, or admit a mistake, individuals perform a tacit calculation of interpersonal risk. Often people will not speak up. Such self-protection may have an adverse impact on patient care and may as well contribute to physician burn-out [4]. Ultimately, an epidemic of silence will overtake the witnesses of the consequences of interpersonal risk taking [1]. The negative impact of such silence is that it suppresses efforts aimed at maintaining a safe patient and trainee environment such as identifying work-arounds, failures to adhere to policy, poor knowledge, and near-misses.

The hierarchy of medical training further impedes the willingness of learners to speak up. In the hierarchy of medicine, authoritarianism drives residents' and students' routines, beliefs, and behaviors. The threat or risk that comes from this hierarchy includes disapproval and negative consequences in evaluations causing trainees to perceive the environment as punitive. When facing clinical uncertainty or a situation known to be associated with poor patient outcomes, residents and students often delay care decisions by consulting those who are lower in the professional hierarchy rather than asking the attending physician [5-7].

\section{Psychological safety}

Psychological safety is a feeling that one is comfortable expressing and being themselves, and sharing concerns and mistakes without fear of embarrassment, ridicule, shame, or retribution [1-3]. Psychological safety exists when individuals feel free to take risks, raise problems, disagree, ask questions, and admit mistakes.

Psychological safety is a relatively unknown concept to many clinician-educators, and trainees. However, it has been shown to be the key element of successful teams in business environments and medicine. Psychological safety is critical to work that is complex, uncertain, and ambiguous, all of which characterizes work in the clinical learning environment. Amy Edmondson, $\mathrm{PhD}$, described psychological safety in the early 1990s [3]. Her research demonstrates the key role of psychological safety in work engagement, quality improvement, error reporting, and team learning. Initially, her research studied error reporting in the hospital setting. She had originally hypothesized that high functioning teams with better communication would have lower error rates. To the contrary, she discovered that high functioning teams reported more errors, because people in these teams talked openly about errors in order to improve and learn from. Highly technical and customized operations are only possible with efficient teamwork, and teamwork is needed to deliver quality patient care in hospital systems. Dr. Edmondson's work established that innovation is driven in organizations with high-performing teams due, in part, to the presence of psychological safety. While data on psychological safety in medical education are limited, emerging evidence shows a strong association between psychological safety and trainee satisfaction with the clinical learning environment $[8,9]$.

\section{Vulnerability in cognitive apprenticeship: the formal vs the hidden curricula}

At this stage of professional development, Dr. Wong and other learners are engaged in a form of cognitive apprenticeship. Traditional apprenticeship refers to a learner being under the tutelage of a master craftsman, a form of on-the job training whereby the apprentice observes and assists the master to the end of proficiency. On the other hand, cognitive apprenticeships are used to teach skills that require internal thought processes and that cannot be readily observed by the teacher [10]. It requires learners to take cues from role models, to be coached by supervisors, and to increase learning by building upon prior information (scaffolding). The tools to accomplish this are often 
metacognitive requiring articulation of thought processes and reflection on ways of solving a problem or completing a task [11]. A transition period occurs whereby students and residents shift from looking from outside in as a lay person and observer, to being personally invested in patients not just as "cases" but as actual suffering individuals with agency [11-13].

Apprenticeship in a clinical setting, as opposed to the preclinical years of medical school, represents learning in a less controlled environment where interpersonal interaction, ambiguity, uncertainty, and taking interpersonal risks are more apparent to the learner $[10,11,14]$. This a type of disorienting dilemma that impels maturation from apprentice to master and that embodies the process of transformational learning. It culminates in a new professional self-identity that is formed by the culture of medicine. The culture of medicine is formed both explicitly through formal didactics and implicitly through a hidden curriculum. The hidden curriculum seeks to imbue trainees with the traits necessary to become leaders in the health care environment. These traits serve as benchmarks for what a physician should be. For example, clinical instructors may emphasize important traits by role modeling intelligence, meticulousness, and conscientiousness. In this way, the hidden curriculum has a powerful effect on shaping medical culture $[15,16]$. Put another way, the formal curriculum is explicit and declared; it outlines the framework and content of knowledge and skills that a trainee is expected to learn-for example, learning about lupus pathophysiology through a lecture. The hidden curriculum, on the other hand, is implicit and refers to the socialization process occurring daily whereby norms and values are transmitted and projected by the teacher. Consciously or not, and the learner takes on the same norms and values $[5,6]$.

However, the hidden curriculum may run afoul of leadership development. A leadership trait in one circumstance may not be constructive in another. Moreover, traits themselves are subjective. For example, "strong" residents are described as being able to handle complex patient situations largely independent of supervision. However, the personality trait of "strength" may not reflect the process competency of "empathy."

While there exist sets of knowledge, skills, and some attitudes and behaviors that are explicitly taught in medicine, the hidden curriculum exerts a powerful influence on professional development. The unspoken ideas and morals of the hidden curriculum influence the development of students and trainees through both positive and negative interactions in clinical settings [7, 8]. Specifically, the hidden curriculum shines when it exerts its positive influence through various means including empathy, resilience, perseverance, and psychological safety. On the other hand, the hidden curriculum exerts its negative influence on trainees and faculty when it fosters lack of accountability to patients, legal phobia, apathy, and the negative effects of work-life balance $[15,16]$.

Rude, dismissive, and aggressive communication among doctors is a part of the culture of medicine [17]. Physician incivility has been shown to impair decision-making, procedural performance, information sharing, help seeking, and to promote demotivation, and emotional exhaustion [5-7, 18-22]. In the clinical learning environment, comments such as "where did you go to medical school," or "are you really a second-year fellow," or "your intern knows more than you do," are the types of communication that decreases willingness to take interpersonal risks. In Dr. Wong's situation, the behavior of the attending teaches learners that to admit one's own limitations and to reach out to others for help is a sign of weakness. The attending's behavior is at the same time coercive. It also conveys that the hierarchy of medicine should be accepted without question.

Historically, medical education culture has viewed shame and guilt as motivators of learning and resilience. While shaming experiences can lead to debilitating emotional and physical reactions including social isolation, disengagement from learning, loss of empathy, unprofessionalism, and impaired learning, it can also function as a disorienting dilemma that ultimately leads to enhanced learning, increased willingness to reach out for help, and improved relationships [20-23]. Nevertheless, shaming and humiliation are not recommended as ways to incentivize learners. These negative aspects of the hidden curriculum essentially teach students and residents to avoid and suppress emotion, and to be defensive and appear apathetic to patients' concerns.

Educators can combat the negative effects of the hidden curriculum [22]. In situations where the hidden curriculum may have already exerted negative effects, educators must encourage communication. Furthermore, the promotion of metacognitive skills such as reflection should be encouraged. Engaging in reflection can have the potential to reorient learners to unmask within themselves the loss of control that was triggered by negative situations. At the same time, reflection preserves professionalism by revealing prejudices and stereotypes [24, 25]. Reflection may also have the potential to promote emotional maturity, relational skills, and patientcentered attitudes [12, 24-26].

\section{Psychological safety and leadership: educators are leaders}

Psychological safety is shaped by leadership [1-3]. Educators take on the "temporary" role of leaders on teams, wards, and within departments. Leaders can promote psychological safety by inviting participation and responding supportively. Setting the stage involves framing and emphasizing the purpose of the work thereby creating shared expectations and meaning. Leaders set the stage by reframing the definition of power from that of coerciveness to that of a shared resource within the health care team. Behaviors such as direction setting, inviting input to clarify and improve the work environment, and creating conditions for continued learning are not unique to so-called strong leaders and are available to 
everyone [2]. In this manner, medical teams may restructure their self-identity as learning laboratories.

Inviting participation is a tool by which educators may overcome the innate tendency towards impression management among learners. Inviting participation serves to clarify the leadership vision, align thinking within the team, and inspire and empower trainees. Two leadership behaviors that promote participation are situational humility and proactive inquiry to learn about an issue, situation, or person [1]. Naïve realism is the term sometimes used to describe the process of making attributions. Attributions are a caesura in the discovery process. In other words, attributions undermine curiosity. With proactive inquiry, genuine questions about the situation convey respect for the person and their contributions to the situation and allow the entire team to learn. To be effective with proactive inquiry, rather than asking yes-no questions or questions for which they already know the answer, leaders must ask question for which they do not have answers. They should ask team members questions that allow them to answer in a focused way. Examples of proactive inquiry might be: "I need your help because I may have missed something" or "what did you see" or "you were here overnight, what did you notice" or "what do you think? I would love your input on this."

A nonpunitive working environment involves thanking and giving credit to others when they offer ideas, provide their input, and ask questions. Empowering learners to effect change involves encouraging learners to continue to ask questions, admit mistakes, and report errors. A sense of personal failure and lack of support threatens the individual with social isolation that may culminate in a state of existential distressed termed demoralization [27]. Such embitterment breeds a sense of helplessness that undermines learning [15, 22]. Inviting learners through acknowledgment and praise are mechanisms employed to create participative leadership [28]. Finally, a psychologically safe environment is one that destigmatizes failure. Debriefing is a form of group reflection that starts with asking, "What did we do well?" and "What can we do better?" It conveys the concept of failure as a natural consequence of uncertainty rather than something that is unacceptable $[2,3]$. In this manner, effective performers learn from and share lessons from failure.

\section{Ambiguity in rheumatology: psychological safety embraces scientific dialog}

In clinical medicine, the context of psychological safety is usually patient safety and quality improvement. However, psychological safety should also be put in the context of scientific dialog. Ambiguity and uncertainty are common occurrences when dealing with the diagnosis and management of rheumatic diseases. The lack of definitive tests or biomarkers and the incomplete understanding of the immunopathology of disease, contribute to the anxieties in making decisions for and with patients. Ambiguity in rheumatology is exacerbated by the fact that experts may at times cannot elucidate how diagnostic and management guidelines should be used in specific and often complex contexts [29]. Another contributing factor to uncertainty and ambiguity in rheumatology is the heterogenous presentations of disease. Ambiguity within rheumatology, per se, spurs an environment that depends on scientific dialog and promotes clinical reasoning [29-31]. To this end, the teacher's ability to question or give feedback determines discourse patterns and the attainment of reasoning skills. Respectful argumentative discourse, or scientific dialog, is one way to promote critical thinking and reasoning [29-31].

\section{Psychological safety in essence}

Amy Edmondson has made the following assertions [1-3] regarding psychological safety: It is not about being nice. It is about candor. It is not a personality factor. It is not the same as trust - trust refers to interactions between two individuals or parties, exists in the mind of an individual, and pertains to a specific entity. Trust is about giving others the benefit of the doubt. Psychological safety, on the other hand, relates to whether others will give you the benefit of the doubt when you have asked for help, or admitted a mistake. Psychological safety is not about lowering performance standards. It is not the absence of conflict. By the same token, diversity is not enough to overcome psychological safety. Psychological safety resides at the group level and may be found in interpersonal relationships.

In conclusion, psychological safety should be an explicit concept known to and embraced by educators. Educators need to acknowledge feelings and emotions triggered by encounters with patients and interactions with colleagues in the workplace. Medical education goes beyond just the attainment of knowledge and skills. It inculcates attitudes, including a compassionate and empathetic approach, that make quality care patient-centered. It is not about getting rid of ambiguity or uncertainty. It is about opening up scientific dialog and providing an environment for argumentative discourse; and it starts with making explicit, the hidden curriculum.

\section{Compliance with ethical standards}

Disclosures None.

\section{References}

1. Edmondson AC (2012) Teaming: how organizations learn, innovate, and compete in the knowledge economy. Jossey-Bass, San Francisco 
2. Edmondson AC (2013) Teaming to innovate. Jossey-Bass, San Francisco

3. Edmondson AC (2018) The fearless organization: creating psychological safety in the workplace for learning, innovation, and growth. Wiley, Hoboken

4. Yigit AO, Ay FA (2019) The effect of emotional labor and impression management on burnout: example of family physicians. Pak $\mathrm{J}$ Med Sci 35(3):793-796. https://doi.org/10.12669/pjms.35.3.4

5. Mahood SC (2011) Beware the hidden curriculum. Can Fam Physician 57(9):983-985

6. Gaufberg EH, Batalden M, Sands R, Bell SK (2010) The hidden curriculum: what can we learn from third-year medical student narrative reflections? Acad Med 85(11): 1710

7. Haidet P, Stein HF (2006) The role of the student-teacher relationship in the formation of physicians: the hidden curriculum as process. J Gen Intern Med 21(1 suppl):S16-S20

8. Torralba KD, Loo LK, Byrne JM, Baz S, Cannon GW, Keitz SA, Wicker AB, Henley SS, Kashner TM (2016) Does psychological safety impact the clinical learning environment for physician residents? Results from the VA's Learners' Perceptions Survey. J Grad Med Educ 8(5):699-707

9. Keitz SA, Aron DC, Brannen JL, Byrne JM, Cannon GW, Clarke CT, Gilman S, Hettler DL, Kaminetzky CP, Wikcer AB, Zeiss RA, Bernett DS (2019) Impact of clinical training on recruiting graduating health professionals. Am J Manag Care 25(4):e111-e118

10. Butler BA, Butler CM, Peabody TD (2019) Cognitive apprenticeship in orthopaedic surgery: updating a classic educational model. J Surg Educ 76(4):931-935

11. Collins A (2014) Cognitive apprenticeship. In: Sawyer R (ed) Cambridge handbook of the learning sciences. Cambridge University Press, Cambridge, pp 47-60

12. Byszewki A, Hendelman W, McGuinty C, Moineau G (2012) Wanted: role models - medical students' perceptions of professionalism. BMC Med Educ 12:115

13. Schel E, Knoop HS, Gismervik MN, Mylopoulos M, Boudreau JD (2019) Stretching the comfort zone: using early clinical contact to influence professional identity formation in medical students. J Med Educ Curric Dev 26(6):2382120519843875

14. Rassie K (2017) The apprenticeship model of clinical medical education: time for structural change. N Z Med J 130:66-72

15. Hafferty FW, Gaufberg EH, O'Donnell JF (2015) The role of the hidden curriculum in "on doctoring" courses. AMA J Ethics 17(2): 129-137

16. Rajput V, Mookerjee A, Cagande C (2017) The contemporary hidden curriculum of medical education. MedEdPublish 6(3):41. https://doi.org/10.15694/mep.2017.000155

17. Pattani R, Ginsburg S, Mascarenhas Johnson A, Moore JE, Jassemi S, Straus SE (2018) Organizational facotrs contributing to incivility at an academic medical center and system-based solutions: a qualitative study. Acad Med 93(10):1569-1575
18. Riskin A, Erez A, Foulk TA, Kugleman A, Gover A, Shoria I, Riskin KS, Bamberger PA (2015) The impact of rudeness on medical team performance: a randomized controlled trial. Pediatrics 136:489-495

19. Riskin A, Erez A, Foulk TA, Riskin-Geuz KS, Ziv A, Sela R, Pessach-Geblum L, Bamberger PA (2017) Rudeness and medial team performance. Pediatrics 139:1-11

20. Bynum WE 4th, Artino AR Jr, Uljtdehaage S, Webb AMB, Varpio L (2019) Sentinel emotional events: the nature, Triggers, and Effects of Shame Experiences in Medical Residents. Acad Med 94(1):85-93

21. Bynum WE 4th, Goodie JL (2014) Shame, guilt, and the medical learner: ignored connections and why we should care. Med Educ 48(11):1045-1054

22. Benbassat $\mathbf{J}$ (2013) Undesirable features of the medical learning environment: a narrative review of the literature. Adv Health Sci Educ Theory Pract 18(3):527-536. https://doi.org/10.1007/s10459012-9389-5

23. Feilchenfeld Z, Kuper A (2018) Passion projects and disorienting dilemmas. Perspect Med Educ 7(5):290-291

24. Hayton A, Kang I, Wong R, Loo LK (2015) Teaching medical students to reflect more deeply. Teach Learn Med 27(4):410-416

25. Borgstrom E, Morris R, Wood D, Cohn S, Barclay S (2016) Learning to care: medical students' reported value and evaluation of palliative care teaching involving meeting patients and reflective writing. BMC Med Educ 16(1):306

26. Domen RE (2016) The ethics of ambiguity: rethinking the role and importance of uncertainty in medical education and practice. Acad Pathol 3:2374289516654712. https://doi.org/10.1177/ 2374289516654712

27. Nanni MG, Caruso R, Sabato S, Grassi L (2018) Demoralization and embitterment. Psychol Trauma 10(1):14-21. https://doi.org/10. $1037 /$ tra0000326

28. Ferraris VA (2015) "Lead from the front": participative leadership. J Thorac Cardiovasc Surg 150(6):1413-1415. https://doi.org/10. 1016/j.jtcvs.2015.09.076

29. Lee SC, Irving KE (2018) Development of Two-Dimensional Classroom Discourse Analysis Tool (CDAT): scientific reasoning and dialog patterns in the secondary science classes. Int J STEM Educ 5:5. https://doi.org/10.1186/s40594-018-0100-0

30. Felton M, Garcia-Mila M, Villarroel C, Gilabert S (2015) Arguing collaboratively: argumentative discourse types and their potential for knowledge building. Br J Educ Psych 85:372-386

31. Osbourne J (2010) Arguing to learn in science: the role of collaborative, critical discourse. Science 328(5977):463-466. https://doi. org/10.1126/science.1183944

Publisher's note Springer Nature remains neutral with regard to jurisdictional claims in published maps and institutional affiliations. 